\title{
UPPER SEMICONTINUOUS DIFFERENTIAL INCLUSIONS WITHOUT CONVEXITY
}

\author{
A. BRESSAN, A. CELLINA AND G. COLOMBO
}

(Communicated by Kenneth R. Meyer)

\begin{abstract}
We prove existence of solutions to the Cauchy problem for the differential inclusion $\dot{x} \in A(x)$, when $A$ is cyclically monotone and upper semicontinuous.
\end{abstract}

\section{INTRODUCTION}

In this paper we deal with the problem of existence of absolutely continuous solutions to differential inclusions with a right-hand side $F$ upper semicontinuous. For this class of inclusions, it is well known that existence holds under the additional assumption of convexity of the values of $F$ (see for instance the chapter 2 of [1]), while it is easy to give counterexamples to the existence of solutions when the assumption of convexity is dropped.

The simplest example of a differential inclusion with upper semicontinuous right-hand side such that the Cauchy problem

$$
\dot{x}(t) \in-F(x(t)), \quad x(0)=0, t \geq 0,
$$

has no solutions is given by the monotonic map $F$ defined as

$$
F(x)= \begin{cases}+1 & x>0 \\ \{-1,+1\} & x=0 \\ -1 & x<0 .\end{cases}
$$

We remark that the above map $F$, although monotone, is not maximal, since the values are not convex. For the same $F$, the problem

$$
\dot{x}(t) \in F(x(t)), \quad x(0)=0, t \geq 0,
$$

has exactly two solutions, namely $x_{1}=t$ and $x_{2}=-t$. Hence this is an example of a differential inclusion with an upper semicontinuous, nonconvex valued right-hand side such that the corresponding Cauchy problem has a closed nonempty set of solutions.

Received by the editors July 20, 1988 and, in revised form, December 2, 1988.

1980 Mathematics Subject Classification (1985 Revision). Primary 34A60.

The first author is on leave from the University of Colorado, Boulder. 
From another point of view, consider any closed set $K \subseteq \mathbf{R}^{n}$, and the projector of best approximation on $K$ from $x, \pi_{K}(x)$,

$$
\pi_{K}(x)=\{y \in K: d(x, y)=d(x, K)\}
$$

In the special case of $X=\mathbf{R}$ and $K=\{-1,+1\}$, example (2) is the problem

$$
\dot{x}(t) \in \pi_{K}(x(t)), x(0)=0 \text {. }
$$

Purpose of the present note is to show that existence of solutions holds in general for any Cauchy problem of the form

$$
\dot{x}(t) \in A(x(t)), \quad x(0)=\xi \in \mathbf{R}^{n},
$$

with $A$ an upper semicontinuous, cyclically monotone map with closed nonempty values.

The map $x \rightarrow \pi_{K}(x)$ affords an example of such an operator.

The argument used in the proof is based on showing that in the present case the convergence of a sequence of approximate solutions implies the strong convergence of their derivatives.

\section{MaIN Result}

We recall the definition and some properties of a cyclically monotone map.

Definition. A multifunction $A: \mathbf{R}^{n} \rightarrow \mathbf{R}^{n}$ is called cyclically monotone if for every cyclical sequence

$$
x_{0}, x_{1}, \ldots, x_{N}=x_{0} \quad(N \text { arbitrary })
$$

and every sequence $y_{i} \in A\left(x_{i}\right), i=1, \ldots, N$, we have

$$
\sum_{i=1}^{N}\left\langle x_{i}-x_{i-1}, y_{i}\right\rangle \geq 0 \text {. }
$$

Proposition 1. [2, Theorem 2.5, p. 38] $A$ is cyclically monotone if and only if there exists a proper convex lower semicontinuous function $V: \mathbf{R}^{n} \rightarrow \mathbf{R}$ such that

where $\partial V$ is the subdifferential of $V$.

$$
A(x) \subseteq \partial V(x)
$$

We denote by $B$ the open unit ball of $\mathbf{R}^{n}$. A map $A$ is called upper semicontinuous if for every $x$ and every $\varepsilon>0$ there exists $\delta>0$ such that $x^{\prime}$ in $x+\delta B$ implies $A\left(x^{\prime}\right) \subset A(x)+\varepsilon B$. Recall that an upper semicontinuous map with closed values has closed graph.

The following is our main result.

Theorem. Let $A$ be a map from $\mathbf{R}^{n}$ into the compact nonempty subsets of $\mathbf{R}^{n}$, upper semicontinuous and cyclically monotone. Then there exists $\delta>0$ such that on $[0, \delta]$ the Cauchy problem

$$
\dot{x} \in A(x), \quad x(0)=\xi,
$$

admits a nonempty closed set of solutions. 
Proof. By Proposition 1 there exists a proper lower semicontinuous convex function $V$ such that $A(x) \subset \partial V(x)$. Since $A$ is locally bounded (see [1, Proposition 1.1.3]), the same holds for $\partial V$. In fact, suppose that for every $x$ in some open set $U$ we have that $\sup \{|y|: y \in A(x)\}$ is bounded by $M>0$ and assume, by contradiction, that there exist $x^{*} \in U$ and $y^{*} \in \partial V\left(x^{*}\right)$ such that $\left|y^{*}\right|>M$. For a sufficiently small positive $\lambda$, the point $x=x^{*}+\lambda y^{*}$ belongs to $U$. Choose $y \in A(x)$. Then

$$
\left\langle y-y^{*}, x-x^{*}\right\rangle=\lambda\left\langle y-y^{*}, y^{*}\right\rangle<0,
$$

which contradicts the monotonicity of the multifunction $\partial V$. Hence we can assume that there exists an open ball about $\xi, B[\xi, R]$ and a $M<\infty$ such that $V$ is Lipschitz continuous with constant $M$ on $B[\xi, R]$, and $A$ is bounded by $M$ on $B[\xi, R]$. By choosing $\delta$ less that $R / M$ we have that no Lipschitzian function $x$ with Lipschitz constant $M$ and such that $x(0)=\xi$ can leave $B[\xi, R]$ on $[0, \delta]$.

Our purpose is to define on $[0, \delta]$ a family of polygonals and to show that a subsequence converges to a solution to $(\mathrm{CP})$. Define the $n$th polygonal by setting

$$
\begin{aligned}
& x_{n}(0)=\xi, \\
& x_{n}\left((i+1) \frac{\delta}{n}\right)=x_{n}\left(i \frac{\delta}{n}\right)+\frac{\delta}{n} y_{i}, \quad i=0, \ldots, n-1,
\end{aligned}
$$

where $y_{i}$ belongs to $A\left(x_{n}(i \delta / n)\right)$, and linearly between the nodal points $i \delta / n$, $(i+1) \delta / n$. The $x_{n}$ are Lipschitzian with Lipschitz constant $M$. The sequence of pairs $\left(\left(x_{n}, \dot{x}_{n}\right)\right)_{n}$ is precompact in $C \times L^{2}$, the first space with the sup norm and the second with the weak topology. Consider a subsequence (that we denote with the same indexes) converging to $(x, \dot{x})$.

We claim that $\|\dot{x}\|_{2}=\lim \left\|\dot{x}_{n}\right\|_{2}$, so that $\dot{x}_{n}$ converges to $\dot{x}$ in $L^{2}$-norm $[3$, p. 124].

Let us remark that, from known results (see [1, Theorem 1.4.1]), $x$ is a solution to

$$
\dot{x}(t) \in \operatorname{co} A(x(t)) \subseteq \partial V(x(t)), \quad x(0)=\xi .
$$

Both the maps $t \rightarrow x(t)$ and $t \rightarrow V(x(t))$ are Lipschitzian, hence differentiable a.e. By Lemma 3.3 in $[2$, p. 73$]$,

By integrating

$$
\frac{d}{d t}(V(x(t)))=|\dot{x}(t)|^{2} \text { a.e. on }[0, \delta] .
$$

$$
V(x(\delta))-V(\xi)=\int_{0}^{\delta}|\dot{x}(\tau)|^{2} d \tau .
$$

On the other hand, for each polygonal line on each interval $(i \delta / n,(i+1) \delta / n)$, the convexity of $V$ implies

$$
V\left(x_{n}\left((i+1) \frac{\delta}{n}\right)\right) \geq V\left(x_{n}\left(i \frac{\delta}{n}\right)\right)+\left\langle\dot{x}_{n}(t) \frac{\delta}{n}, y\right\rangle
$$


for every $y$ in $\partial V\left(x_{n}(i \delta / n)\right)$; hence in particular, for $y \equiv \dot{x}_{n}$ on each $(i \delta / n$, $(i+1) \delta / n)$,

$$
V\left(x_{n}(\delta)\right)-V(\xi) \geq \int_{0}^{\delta}\left|\dot{x}_{n}(\tau)\right|^{2} d \tau .
$$

By passing to the limit for $n \rightarrow \infty$ and using the continuity of $V$ at the point $x(\delta)$,

$$
\limsup \int_{0}^{\delta}\left|\dot{x}_{n}(\tau)\right|^{2} d \tau \leq \int_{0}^{\delta}|\dot{x}(\tau)|^{2} d \tau .
$$

By the weak lower semicontinuity of the norm, we have that

$$
\liminf \int_{0}^{\delta}\left|\dot{x}_{n}(\tau)\right|^{2} d \tau \geq \int_{0}^{\delta}|\dot{x}(\tau)|^{2} d \tau,
$$

so that the claim is proved.

A subsequence of $\dot{x}_{n}$ converges pointwise almost everywhere.

By our construction,

$$
d\left(\left(x_{n}(t), \dot{x}_{n}(t)\right), \operatorname{graph}(A)\right) \leq \frac{\delta}{n} M
$$

since $\operatorname{graph}(A)$ is closed and, on the complement of a null set, $\left(x_{n}(t), \dot{x}_{n}(t)\right)$ converges to $(x(t), \dot{x}(t))$,

$$
\dot{x}(t) \in A(x(t)) \text { a.e. }
$$

This proves that the set of solutions to $(\mathrm{CP})$ is nonempty. Let $\left(y_{m}\right)$ be solutions converging to $y$ in $C([0, \delta])$. By taking a subsequence, we can assume that $\left(\dot{y}_{m}\right)$ converges weakly in $L^{2}$. We apply (4) directly to $y_{m}$ and to $y$ to obtain that $\dot{y}_{m}$ converges to $\dot{y}$ in the norm topology of $L^{2}$. The same argument as before shows that $y$ is a solution to (CP). Hence, the set of solutions to (CP) is closed in $C([0, \delta])$.

\section{AN APPLICATION}

Proposition 2. Let $K$ be a closed nonempty subset of $\mathbf{R}^{n}$ with the Euclidean norm and define the projection $\pi_{K}$ as in (3). Then there exists a convex function $V: \mathbf{R}^{n} \rightarrow \mathbf{R}$ such that

$$
\pi_{K}(x) \subseteq \partial V(x), \quad \forall x \in \mathbf{R}^{n} .
$$

Proof. For every $u \in \mathbf{R}^{n}$, consider the functional

$$
\varphi_{u}(x)=\frac{1}{2}|u|^{2}+\langle u, x-u\rangle,
$$

whose graph is the hyperplane tangent to the graph of $x \rightarrow|x|^{2} / 2$ at the point $\left(u, \frac{1}{2}|u|^{2}\right)$. Observe that, for every $x, u, v \in \mathbf{R}^{n}, u \neq v$, one has

$$
|x-u| \leq|x-v| \text { iff } \varphi_{u}(x) \geq \varphi_{v}(x) \text {. }
$$

Indeed, the set $H=\left\{x ; \varphi_{u}(x)=\varphi_{v}(x)\right\}$ is the affine hyperplane

$$
\left\{x \in \mathbf{R}^{n}:\langle u-v, x\rangle=\frac{1}{2}\left(|u|^{2}-|v|^{2}\right)\right\}
$$


which is orthogonal to $u-v$. Moreover, the midpoint $(u+v) / 2$ of the segment joining $u$ and $v$ lies on $H$. Therefore $\varphi_{u}(x)=\varphi_{v}(x)$ iff $|x-u|=|x-v|$. Since $\varphi_{u}(u) \geq \varphi_{v}(u)$, the linearity of $\varphi_{u}$ and $\varphi_{v}$ implies (5). After these preliminaries, define

$$
V(x)=\sup \left\{\varphi_{u}(x): u \in K\right\} .
$$

Clearly $V$ is convex, everywhere defined and locally bounded. More precisely:

$$
V(x) \leq \frac{1}{2}|x|^{2}=\sup \left\{\varphi_{u}(x): u \in \mathbf{R}^{n}\right\} .
$$

In order to prove that $\pi_{K}(x) \subseteq \partial V(x)$, for every $u \in \pi_{K}(x)$ it suffices to show that $V(x)=\varphi_{u}(x)$, i.e. $\varphi_{v}(x) \leq \varphi_{u}(x)$ for every $v \in K$. Since $|u-x| \leq|v-x|$, this is a consequence of $(5)$.

Corollary. Let $K \subseteq \mathbf{R}^{n}$ be closed. Then the Cauchy problem

$$
\dot{x} \in \pi_{K}(x), \quad x(0)=\xi,
$$

admits a closed nonempty set of solutions defined on $[0,+\infty)$.

Proof. Combining our main Theorem with Proposition 2, one obtains the local existence of forward solutions. Since there exist constants $a$ and $b$ such that

$$
|y| \leq a|x|+b \quad \text { for every } y \in \pi_{K}(x),
$$

every local solution admits an extension to $[0,+\infty)$.

\section{REFERENCES}

1. J. P. Aubin and A. Cellina, Differential inclusions, Springer-Verlag, Berlin, 1984.

2. H. Brézis, Opérateurs maximaux monotones et semi-groupes de contractions dans les espaces de Hilbert, North-Holland, Amsterdam, 1973.

3. K. Yosida, Functional analysis, 6th edn. Springer-Verlag, Berlin, 1980.

SisSa, V. le Miramare, 11, I=34014 Trieste, Italy 